\title{
La enseñanza de la física a través de los juguetes
}

Giorgio Häusermann

\begin{abstract}
Uno de los aspectos más difíciles de la enseñanza de la física es ayudar a los estudiantes a reconocer sus aplicaciones. A menudo, los alumnos terminan memorizando ecuaciones y métodos, y se descuida la realización de las necesarias prácticas de laboratorio. Cualquier conexión entre las tareas y el "mundo real" rara vez va más allá del aula, y suele morir después del examen.
\end{abstract}

(Skrutvold, 1997).

Recibido: 18 de octubre de 2012 / Aceptado: 20 de enero de 2013

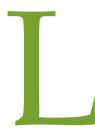
a introducción de losjuguetes en las actividades didácticas permite a los estudiantes construir activamente, con ejemplos concretos, el propio conocimiento en el ámbito de la física. Los juguetes son objetos del mundo real que estimulan el interés y la emotividad. Las experiencias sencillas y los juguetes permiten a todos los estudiantes dar un primer paso hacia el desarrollo de la curiosidad por el saber científico. Escogiendo adecuadamente los juguetes y las experiencias, se pueden examinar desde diversos ángulos, fenómenos físicos que normalmente se proponen de manera lineal, según un planteamiento tradicional del estudio de la física que no permite una visión global que se adecúe mejor a la realidad, tal como la perciben los estudiantes. Cabe destacar que si se pretende concretar nuestra propuesta en actividades didácticas, más allá de la utilización de los juguetes, son necesarios otros recursos didácticos que permitan a los estudiantes interactuar con éstos y aprehender las competencias generales y específicas del campo científico, requeridas por los diversos programas nacionales e internacionales.

Este artículo tiene como objetivo suscitar una reflexión sobre la elección de los juguetes y de las experiencias sencillas que utilizamos en las presentaciones que, desde hace años, venimos haciendo en escuelas, cursos de formación y para público de cualquier edad.

El artículo recoge, a grandes rasgos, las actividades presentadas durante el curso escolar 2008/2009, tanto en una veintena de presentaciones para alumnado de enseñanza primaria y secundaria como en cursos para docentes impartidos en Suiza, Italia y España. Está previsto un segundo artículo que, a partir de las experiencias llevadas a cabo en el aula por nuestro grupo, muestre cómo se pueden utilizar los juguetes

\footnotetext{
* Filosofo, educador, Secretario Permanente de la Comisión Nacional de la UNESCO en Nicaragua.
}

\section{RESUMEN}

Pompas de jabón iridiscentes, globos de colores, pistolas que disparan trocitos de patata, una máquina de humo ... ¿Puede la magia explicar la ciencia? ¿Podemos divertirnos aprendiendo? ¿Podemos construir nuestro propio conocimiento por medio de los juegos? Este artículo pretende dar una respuesta afirmativa a todas estas preguntas, utilizando juguetes de nuestro entorno cotidiano y experiencias sencillas para explicar distintos fenómenos físicos y hacernos reflexionar sobre el mundo que nos rodea.

Palabras clave: ciencia-espectáculo, juegos, emociones positivas, reflexión, competencias.

\section{ABSTRACT}

Iridescent soap bubbles, coloured balloons, shooting bits of potatoes, smoke machines... Can magic explain science? Can we have fun learning? Can we build our own knowledge through games? This article aims to answer yes to all these questions by using common games from all around us and simple experience to explain different physical phenomena and make us think about the world around us.

Key words: Science-show, games, positive emotions, reflection, competences. 
en el contexto de una propuesta didáctica innovadora de la enseñanza de la física.

\section{Los orígenes}

Personalmente descubrimos el uso sistemático de los juguetes con una función didáctica a mediados de los años noventa con la exposición itinerante I Giocattoli e la scienza, organizada por el profesor Vittorio Zanetti (1993). Al observar el interés de los jóvenes visitantes y el entusiasmo de los estudiantes que presentaban la exposición, tuve la oportunidad de comprobar que este tipo de actividades podía ser un puente entre mundos tan distintos como la física, los jóvenes y los objetos de uso cotidiano.

Algunas etapas importantes han sido sucesivamente, los convenios y los seminarios organizados por el GIREP (www.girep.org/), en particular los de Duisburgo de 1998 (Hands-On Experiments in Physics Education) y de Lubiana de 2005 (Informal Learning and Public Understanding of Physics). Una etapa posterior en el desarrollo de la enseñanza no tradicional de la física estuvo caracterizada por la colaboración con Paola Rodari y Giovanni Panizon en la instalación de los objetos expuestos en el Museo del Bali (www.museodelbali.org) en las Marcas. En este caso, un logro importante fue el paso de la utilización del juego con fines didácticos al mundo mucho más fascinante de la educación científica no tradicional, que ya se ha convertido en un elemento formativo insustituible en la sociedad del siglo XXI.

Prueba de ello son los numerosísimos Science Centres que caracterizan la nueva tendencia museística de todos los países y que tienen como objetivo alcanzar un desarrollo sostenible y una calidad de vida que permitan mirar al futuro con esperanza. Otro paso importante ha sido la participación en el Science on Stage del 2005, en el CERN de Ginebra, donde la "Caja de Einstein", tuvo la oportunidad de compararse con las numerosas propuestas presentadas por colegas de muchos países.

Finalmente, cabe recordar que para encontrar los objetos y las experiencias correctas debe buscarse, por ejemplo, en internet, en los catálogos (www.teachersource. com) y las páginas web (www.stevespanglerscience. com) dedicados a este tipo de actividades, y luego experimentar, reflexionar y volver a experimentar.

\section{La lección-espectáculo}

Una de las actividades que proponemos más frecuentemente es la lección-espectáculo presentada bajo títulos diversos como "Aprender la física jugando", "Juega con la física" o "Los juegos de la caja de Einstein". La actividad consiste en recorrer los principales capítulos de una manual de física imaginario, en el cual, en lugar de las páginas, las fórmulas, las definiciones y los ejercicios, hay juguetes y experiencias sencillas que muestran cómo, a través de la observación y reflexión sobre su funcionamiento, se pueden reencontrar los conceptos y los fenómenos estudiados en los cursos de física.

El inicio de la actividad se dedica, generalmente, a estimular al público con una experiencia de "magia". En ella, se vierte agua en un vaso que contiene poliacrilato de sodio. Absorbida por esta sustancia, el agua se adhiere a las paredes del vaso, de modo que éste puede ponerse boca abajo sin que se vierta el agua, con lo que se crea la ilusión de que ésta ha desaparecido. Esta primera experiencia pretende llamar la atención de los espectadores sobre el hecho de que, a menudo tenga un determinado comportamiento, pero en realidad, por una causa concreta desconocida, se comporta de un modo imprevisto.

\section{La magia de la gravitación}

A la pregunta "¿Cuál de entre dos objetos con la misma forma y distinta masa llega antes al suelo, si se dejan caer desde la misma altura?", la mayor parte de los presentes responde que llega antes el de más peso. La finalidad de esta parte de la lección es mostrar que todos los objetos que son lanzados tienen un comportamiento análogo en lo que respecta a la aceleración de la gravedad. En efecto, para todos los lanzamientos, según el ángulo y el módulo del vector de la velocidad inicial, y dejando de lado el rozamiento con el aire, se obtienen trayectorias diferentes, pero con la característica común de ser arcos de una parábola. Un objetivo posterior es identificar las diferentes 
formas de lanzamiento de cada proyectil (presión del aire, fuerza elástica, reacción química, lanzamiento con la mano, etc.) y las características de las transformaciones de energía que producen el movimiento.

Para hacer más espectacular esta parte de la presentación, utilizamos el Airzooca, comprimiendo el bazuca, que lanza una masa de aire hacia el objetivo al que hemos apuntado. En relación con este lanzamiento, se puede decidir si el aire se trata de una masa o si se transmite una vibración parecida a una onda sonora. Tras observar que el tiempo transcurrido entre el disparo y el efecto a distancia es demasiado grande para deberse a una vibración sonora, se concluye que se trata de una masa de aire. Pero ¿de qué forma? Con la ayuda de humo, producido por una máquina de discoteca, hemos verificado que se trata de anillos que se mueven en línea recta. El cuadro 1, recoge los diferentes objetos o instrumentos que se utilizan (imagen 1).

\begin{tabular}{|l|l|l|}
\multicolumn{1}{|c|}{ Objeto } & $\begin{array}{r}\text { Fenómeno específico además del } \\
\text { efecto de la gravitación }\end{array}$ & \multicolumn{1}{c|}{ Referencia } \\
\hline 1a) Cohete de aire. & Presión del aire, rozamiento. & Pump Rockets w/Pump (www.teachersource.com). \\
\hline 1b) Lance con elastic. & Fuerza elastica, momento angular. & X-Zylo Launcher (www.teachersource.com). \\
\hline 1c) Pistola de patata. & Presión del aire. & $\begin{array}{l}\text { Potato Gun } \\
\text { (www.xump.com/Science/PotatoGun.cfm). }\end{array}$ \\
\hline 1d) Cohete de CO2 & $\begin{array}{l}\text { Reacción química, principio de } \\
\text { indeterminación. }\end{array}$ & $\begin{array}{l}\text { Mighty Seltzer Rocket } \\
\text { (www.teachersource.com). }\end{array}$ \\
\hline 1e) Lance de la cuerda. & Trabajo, energía potencial. & String Launcher (www.teachersource.com). \\
\hline 1f) Bazoca de aire. & Onda de presión. & Airzooka (www.teachersource.com). \\
\hline 1g) Pistola de Volta. & Gas, electrostática. & $\begin{array}{l}\text { Piezo-electric Igniter } \\
\text { (www.teacherspirce.com). }\end{array}$ \\
\hline
\end{tabular}

Cuadro 1. Objetos que se utilizan para observar el efecto de la gravitación.

\section{¿Cómo cae y cómo rebota?}

Esta parte pretende mostrar algunos aspectos de la caída libre y de los choques elásticos e inelásticos. Es importante rebatir la creencia de que los objetos de distinta masa pueden llegar juntos al suelo. Con este propósito, además de dos pelotitas de distinto tamaño y masa, se observa el comportamiento en caída libre de un libro y de un folio (normal, arrugado, encima y debajo del libro). A continuación, homogéneo y, por lo tanto, rebota de un modo inesperado. Otros objetivos de esta parte son la observación de la conservación de la energía y el comportamiento de los cuerpos durante la caída libre (imagen 2, cuadro 2).

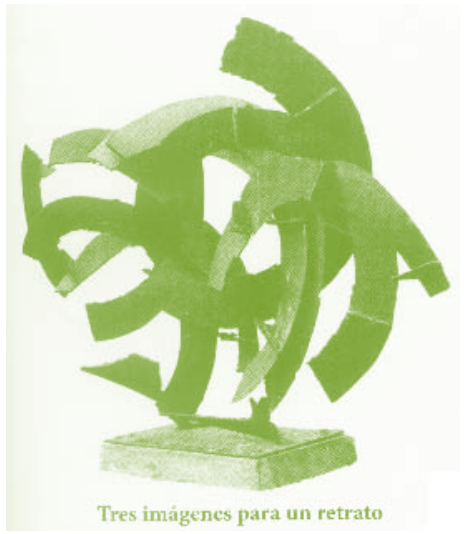

\begin{tabular}{|l|l|l|}
\multicolumn{2}{|c}{ Objeto } & $\begin{array}{c}\text { Fenómeno específico además del } \\
\text { efecto de la gravitación }\end{array}$ \\
\hline \multicolumn{1}{|c|}{ Libro y folio } & \multicolumn{1}{c|}{ Caída con y sin rozamiento. } & \multicolumn{1}{c|}{ Referencia } \\
\hline 1a) Cohete de aire. & Presión del aire, rozamiento. & Pump Rockets w/Pump (www.teachersource.com). \\
\hline 1b) Lance con elastic. & Fuerza elastica, momento angular. & X-Zylo Launcher (www.teachersource.com). \\
\hline
\end{tabular}




\section{Aire, presión y globos}

Existen muchas experiencias que se pueden hacer con el aire, el agua y la presión. Para nuestra presentación hemos escogido algunas que nos parecen especialmente curiosas. Empezamos con la pipa que mantiene en el aire una pelotita ligera, continuamos con el secador de pelo $\mathrm{y}$, finalmente, cogemos un soplador de jardín y mantenemos suspendido un balón hinchable. La salida del aire puede, además, desenrollar velozmente un rollo de papel higiénico, el movimiento del cual durante el lanzamiento describe una parábola. Las pompas de jabón son como globos que no caen de golpe al suelo y permiten que se observe su iridiscencia. Otras experiencias que proponemos son: el globo pinchado con un palito de madera (elasticidad), lleno de agua, que, colocado sobre una vela encendida no explota, y dos globos hinchados de modo diferente que, cuando se atan entre ellos, demuestran que el más pequeño se deshincha a pesar de la idea de los vasos comunicantes.

Como última experiencia, una persona del público hinchará un saco tubular de varios metros de largo. Si se introduce directamente el aire de los pulmones, sólo se hincha una pequeña parte del tubo, mientras que si se sopla a una cierta distancia se hincha casi completamente, ya que se produce una depresión en la embocadura del tubo que permite por la presión atmosférica que el aire fluya hacia el interior.

\begin{tabular}{|c|c|c|}
\hline Objeto & Fenómeno específico & Referencia \\
\hline 3a) Pelotita y phön. & Dinámica de los fluidos. & $\begin{array}{c}\text { Gioca con la física - fluidi } \\
\text { (www.aspti.ch/giocafisica/pagine/giochi.html) } \\
\text { Floating Balls \& Flying Toiler Paper } \\
\text { (www.stevespanglerscience.com/experiment/035). }\end{array}$ \\
\hline 3b) Dos globos atados entre sí. & Presión del aire. & Two Balloon Surprise (www.teachersource.com). \\
\hline 3c) Globo con agua. & Calor específico del agua. & $\begin{array}{l}\text { Fire Water Coolest Conductor of Heat } \\
\text { (www.stevespanglerscience.com/experiments/031). }\end{array}$ \\
\hline 3d) Tubo de aire. & Dinámica de los fluidos. & WindTubes (www.teachersource.com). \\
\hline
\end{tabular}

Cuadro 3. Descripción de los objetos para los experimentos de aire y presión.

\section{Ondas, sonidos y luz}

Los principales objetivos de esta parte son el concepto de onda, transmisión de energía sin transmisión de materia y las propiedades de los instrumentos musicales. Comenzamos siempre observando el comportamiento de los impulsos transmitidos por un muelle largo; esta fase nos permite observar los dos tipos de impulsos, transversales y longitudinales, la dependencia de la velocidad respecto a la tensión, la reflexión y la superposición. A continuación demostramos que los impulsos repetidos en el tiempo producen ondas estacionarias de características diferentes según su frecuencia. Un cilindro de cartón acoplado en un extremo del muelle nos permite "escuchar" los impulsos, transformando en sonido la vibración del muelle.
Éste es el punto de partida para mostrar distintos sonidos producidos por instrumentos musicales que tiene en común la capacidad de hacer vibrar membranas, metales y otros cuerpos, de amplificar las vibraciones y de transmitirlas a través del aire. Hablar de ondas significa también hablar de luz. Además de dos aspectos como la difracción y la composición de los colores, proponemos al público una experiencia que implica la capacidad del cerebro de invertir las imágenes que se forman en la retina. Las lentes que invierten las imágenes verticalmente son un modo divertido para reflexionar sobre el sistema óptico humano y sobre cómo se comporta el cerebro en caso de que éste sufra un trastorno (cuadro 4). 


\begin{tabular}{|c|c|c|}
\hline Objeto & Fenómeno específico & Referencia \\
\hline 4a) Muelle largo. & Propiedades de las ondas & Wave Modeling Spring (www.teachersource.com) \\
\hline 4b) Tono. & Vibración de la membrana. & Thunder Drums (www.teachersource.com). \\
\hline 4c) Barras sonoras. & Vibración metálica. & Energy Chimes (www.teachersource,com) \\
\hline 4d) Cuerno sonoro. & Vibración metálica. & $\begin{array}{l}\text { Singing Drum Spinner (www.teachersource.com) } \\
\text { Singing Rod (www.teachersource.com) }\end{array}$ \\
\hline 4e) Tubo sonoro & Diferencia de presión. & Sound Tubes (www.teachersource.com) \\
\hline 4f) Carillón & Láminas vibrantes. & Gioca con la física - suoni (http:// sciencekit.com) \\
\hline $\begin{array}{l}\text { 4g) Slide whistle y tubo con } \\
\text { silbato. }\end{array}$ & $\begin{array}{l}\text { Diferencia de longitud del } \\
\text { tubo sonoro. }\end{array}$ & Gioca con la física - suoni (http:// sciencekit.com) \\
\hline $\begin{array}{l}\text { 5a) Lentes con redes } \\
\text { reticulares. }\end{array}$ & Difracción de la luz. & Prism Glasses - Double Axis (www.teachersource.com) \\
\hline 5b) Lentes que invierten. & Sistema óptico del ojo. & Inversion Glasses Investigation (http://sciencekit.com) \\
\hline $\begin{array}{l}\text { 5c) ¿Pelota blanca o de } \\
\text { colores? }\end{array}$ & Composición de los colores. & Mysterious Glowing Ball (www.teachersource.com) \\
\hline
\end{tabular}

Cuadro 4: Descripción de los objetos para los experimentos sobre ondas, sonido y luz.

\section{Conclusiones}

Nuestro programa concluye con el lanzamiento de los globos-cohete (Rocket Balloons, www.teacher-source.com) que sueltan una decena de participantes desde diversos puntos del lugar de la presentación. Es una forma de celebrar alegremente el final de una actividad que quiere ser al mismo tiempo un entretenimiento y una ocasión para aprender y reflexionar sobre el mundo que nos rodea.

Nuestra experiencia hace ya más de diez años que dura y siempre ha sido positiva. No sabemos cuánta ciencia habremos transmitido, pero hemos visto en cada sesión a jóvenes y menos jóvenes estar atentos durante más de una hora y media y disfrutar de la ciencia.

\section{Nota}

“AGRADECIMIENTOS. Este artículo ha sido escrito con la colaboración de Marco Caló, profesor de secundaria de Barbengo (Suiza), Pamela De Lorenzi, maestra de primaria (Suiza), Daniela Martínez, del SES El Vern Lli $\square a ́$ de Vall (Barcelona) y Sonia Rubio, del Instituto Collserola de Barcelona, que forman parte del grupo "La compagnia della scatola di Einstein". Sónia Rubio y Daniela Martínez han hecho la traducción del artículo original en italiano.

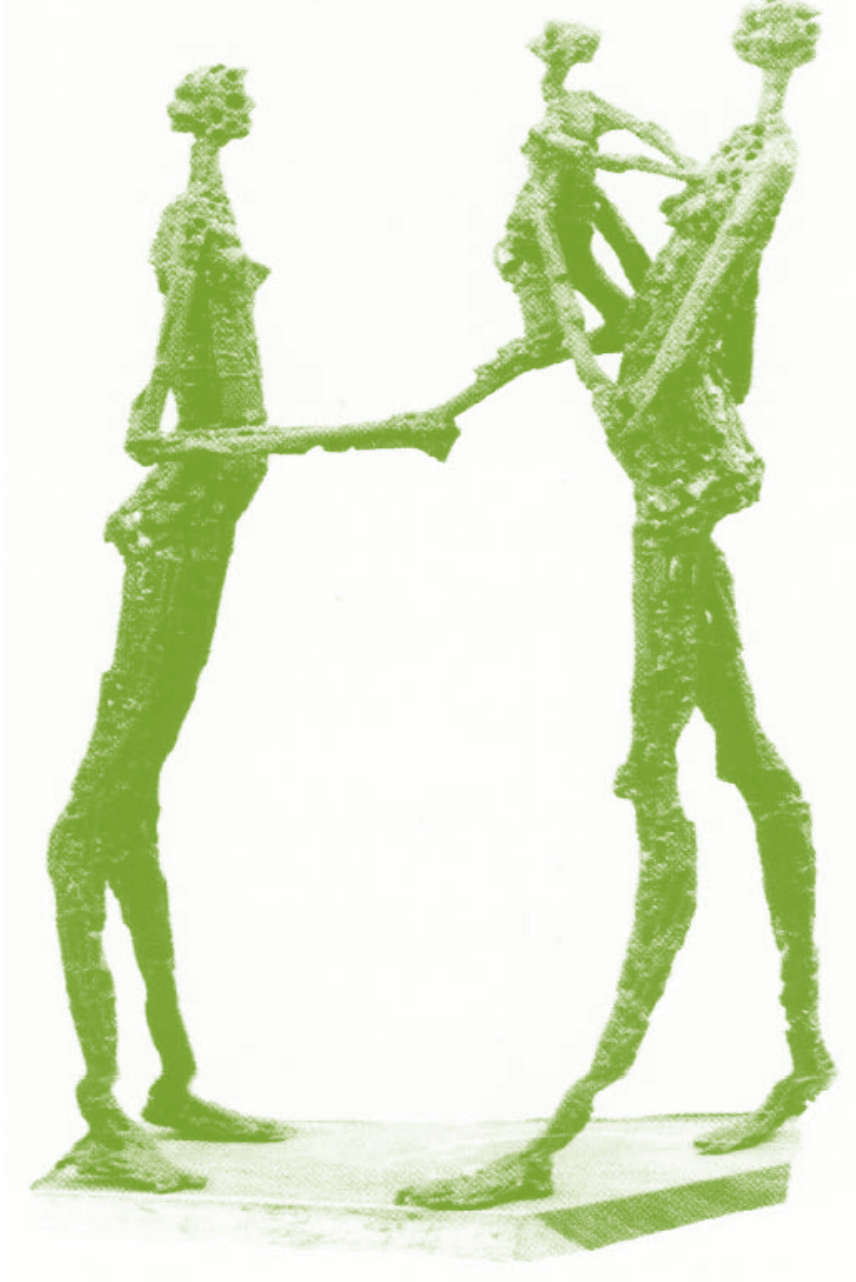




\section{Referencias bibliográficas:}

AA.VV. (1998): Hands on-Experiments in Physics Education Proceeding. ICPE - GIREP International Conference, Duisburg, 23-28 agosto 1998 Deutschland.

FIESSER, L. (1990): Anstiften zum Denken - Die Phänomenta. Flensburg. Pädagogische Hochschule Flensburg Deutschland.

HAEUSERMANN, G. (2009): “Campi di gioco-Il concetto di campo nel funzionamento dei giocattoli". La física nella scuola, Quaderno 19, Associazione per I’Insegnamento della Física, Módena (Italia).

HIXSON, B. K. (2003): Newton Take 3. Loose in the Lab. Inc, Sandy, Utah (EEUU).

SKRUTVOLD, K. (1997): "Playing in Physics. With Traditional Cultura Toys" (en línea) http://members. tripod.com/sharing science/Physics/physicstoys.html.

SPROTT, J.C (2006) Physics Demonstrations, A sourcebook for teachers of physics. The University of Wisconsin Press, Madison, Wisconsin (Estados Unidos).

TAYLOR B. y otros (2005): Teaching Physics with TOYS. Terrific Science Press, Middletown, Ohio (EEUU).

ZANETTI V. (1993): I giocattoli e la scienza. La física nella scuola, Quaderno 4, Associazione per l'Insegnamento dekka Física, Módena (Italia).

\section{Bibliografía web}

Gioca con la física

www.aspti.ch/giocafisica/index.html

Steve Spangler Science

www.stevespanglerscience.com/

Educational Innovations

www.teachersource.com/

XUMP Science Toys www.xump.com/

Science Kit http://sciencekit.com/

Hawkin's Bazaar www.hawkin.com/

Nitinoldrath wwwo.nitinoldraht.del

Supermagnete wwww.supermagnete.ch/

Opitec wwww.opitec.ch/

Grand Illusion: wwww.grand-illusions.com/

\section{Dirección de contacto}

Giorgio Häusermann

Scuola Universitaria Professionale

della Suizzera Italiana (SUPSI). Locarno (Suiza)

ghaeusermann@bluewin.ch

Este artículo fue recibido en ALAMBIQUE. DIDÁCTICA DE LAS CIENCIAS EXPERIMENTALES en enero de 2010 y aceptada en mayo

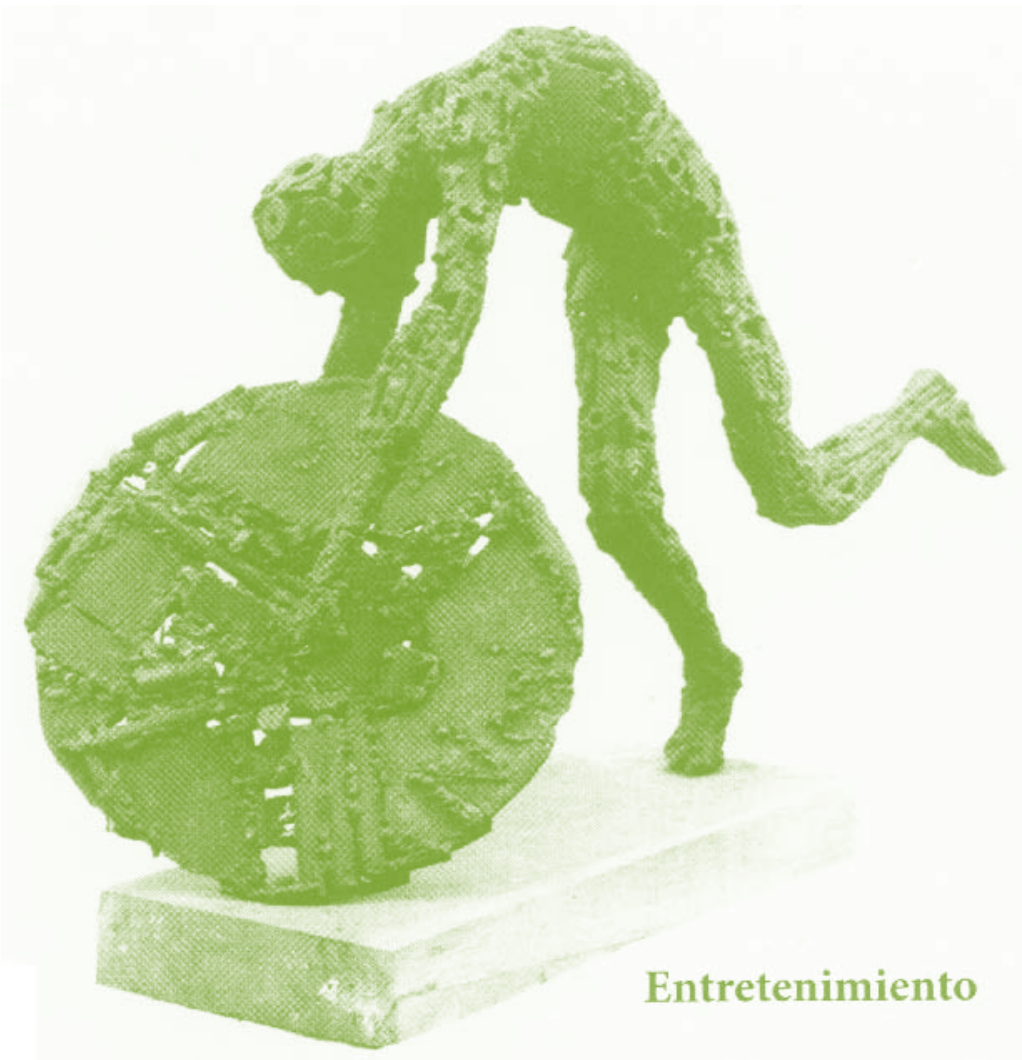

